ఠ

\title{
Axitinib for preoperative downstaging of renal cell carcinoma with sarcomatoid differentiation and direct invasion of the duodenum and inferior vena cava: a case report
}

\author{
This article was published in the following Dove Press journal: \\ OncoTargets and Therapy \\ 15 February 2014 \\ Number of times this article has been viewed
}

\author{
Hideo Yuki ${ }^{1, *}$ \\ Takao Kamai ${ }^{1, *}$ \\ Keiichi Kubota ${ }^{2}$ \\ Hideyuki Abe' \\ Daisaku Nishihara' \\ Tomoya Mizuno' \\ Akinori Masuda' \\ Hironori Betsunoh' \\ Masahiro Yashi' \\ Yoshitatsu Fukabori' \\ Ken-Ichiro Yoshida' \\ 'Department of Urology, \\ ${ }^{2}$ Department of Gastroenterological \\ Surgery, Dokkyo Medical University, \\ Mibu, Tochigi, Japan \\ *These authors contributed equally \\ to this manuscript
}

\begin{abstract}
Background: Renal cell carcinoma (RCC) with sarcomatoid differentiation is invasive, refractory to treatment, and has a higher mortality. Therefore, systemic therapy is still challenging, and the curative resection of localized or locally advanced RCC with sarcomatoid differentiation is very important. Axitinib is a potent and selective second-generation vascular endothelial growth factor receptor tyrosine kinase inhibitor with improved safety and tolerability. Axitinib is generally recommended as second-line therapy for advanced RCC because the phase III axitinib versus sorafenib in advanced RCC (AXIS) trial demonstrated that it achieved longer progression-free survival than sorafenib in patients with metastatic RCC after failure of an approved first-line regimen.
\end{abstract}

Methods: We present a 73-year-old man who had a large (13 $\mathrm{cm}$ in diameter) right RCC with sarcomatoid differentiation that directly invaded the duodenum and inferior vena cava. The patient presented with gastrointestinal bleeding, was unable to eat solid food, and had become emaciated. Thus, his classification was poor risk with anemia, hypercalcemia, and poor performance status, according to the Memorial Sloan-Kettering Cancer Center criteria. He seemed unlikely to survive if radical nephrectomy, cavotomy with thrombectomy, and pancreatoduodenectomy were performed. To reduce the tumor burden and potential operative complications, we administered axitinib as first-line neoadjuvant therapy.

Results: Six weeks of treatment reduced the tumor burden without causing severe toxicities. Subsequently, radical right nephrectomy, cavotomy with thrombectomy, and pancreatoduodenectomy were performed successfully. The pathological treatment effect of axitinib was grade 2 (two-thirds necrosis). The resected tumor showed a heterogeneous reaction for phosphorylated Akt (Ser-473) by Western blotting and immunohistochemistry, indicating that parts of the tumor were sensitive to axitinib and other parts were not.

Conclusion: Axitinib might be promising as preoperative or neoadjuvant therapy for locally advanced $\mathrm{RCC}(>\mathrm{cT} 3 \mathrm{~b}$ or $>\mathrm{cT}$ any $\mathrm{N} 1)$.

Keywords: renal cell carcinoma, sarcomatoid differentiation, axitinib, tyrosine kinase inhibitors, phosphorylated Akt

\section{Introduction}

It is challenging to perform radical nephrectomy and cavotomy plus thrombectomy for locally invasive renal cell carcinoma (RCC) with tumor thrombus. ${ }^{1}$ Before molecular-targeting agents were available, preoperative cytokine therapy was given to a few selected patients with locally advanced RCC, but the value of such therapy was controversial. Recently, several molecular-targeting agents, particularly tyrosine
Department of Urology,

Dokkyo Medical University,

880 Kitakobayashi Mibu,

Tochigi, 32I-0293, Japan

Tel +81282872162

Fax +8I 282867533

Email kamait@dokkyomed.ac.jp 
kinase inhibitors (TKIs), have been employed as neoadjuvant therapy to facilitate subsequent surgery by decreasing the tumor burden, but this has been on an investigational basis and preoperative TKI therapy has not received approval., ${ }^{2,3}$

We treated a man who had locally advanced RCC with sarcomatoid differentiation that showed direct invasion of the duodenum and inferior vena cava. After downsizing the tumor by the administration of axitinib for six weeks, radical right nephrectomy with cavotomy and pancreatoduodenectomy were performed successfully. We discuss the possibility of using neoadjuvant therapy with axitinib to reduce the tumor burden, as was done in this case.

\section{Case report}

In November 2012, a 73-year-old man presented with a right renal mass and the chief complaints of anorexia, fatigue, and melena. Laboratory tests revealed anemia (hemoglobin, $6.2 \mathrm{~g} / \mathrm{dL}$ ) and hypercalcemia (corrected calcium, $11 \mathrm{mg} / \mathrm{dL}$ ). The Karnofsky performance status was 50\%-60\%. Computed tomography (CT) showed a large right renal tumor with a diameter of $13 \mathrm{~cm}$ that had invaded the second part of the duodenum and had involved multiple regional lymph nodes without distant metastasis (Figure 1).

Positron emission tomography (PET) scans showed accumulation of fluorine-18-deoxyglucose in the tumor, with the maximum standardized uptake value being $>40$ (Figure 2). Esophagogastroduodenoscopy detected direct invasion of the tumor into the second part of the duodenum with bleeding (Figure 3). Biopsy of the protruding tumor revealed histologic features that were highly suggestive of sarcoma (Figure 4). Accordingly, the clinical diagnosis was right RCC with

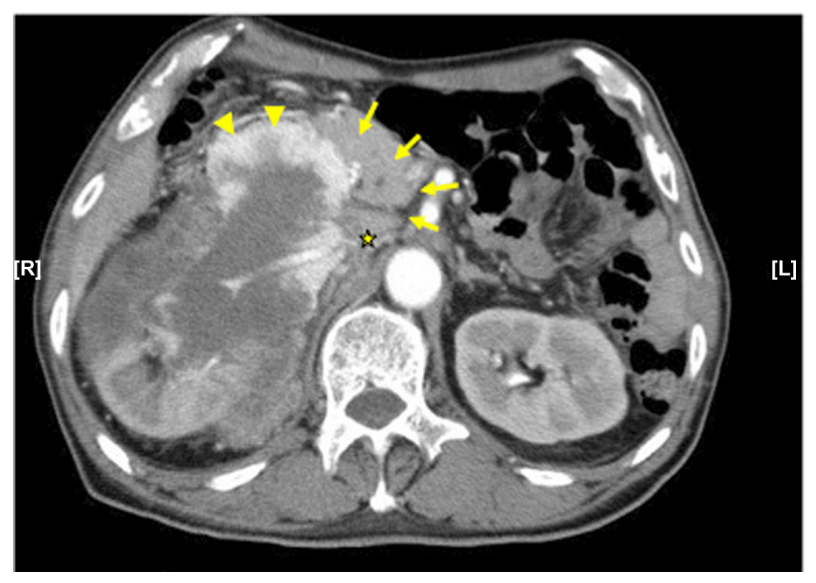

Figure I Enhanced CT.

Notes: This enhanced CT shows a $13 \mathrm{~cm}$ right renal tumor that invades the pancreas (arrows), duodenum (arrowheads), and inferior vena cava (asterisk). The second part of the duodenum shows stenosis because of the protruding right renal tumor. Abbreviation: CT, computed tomography.

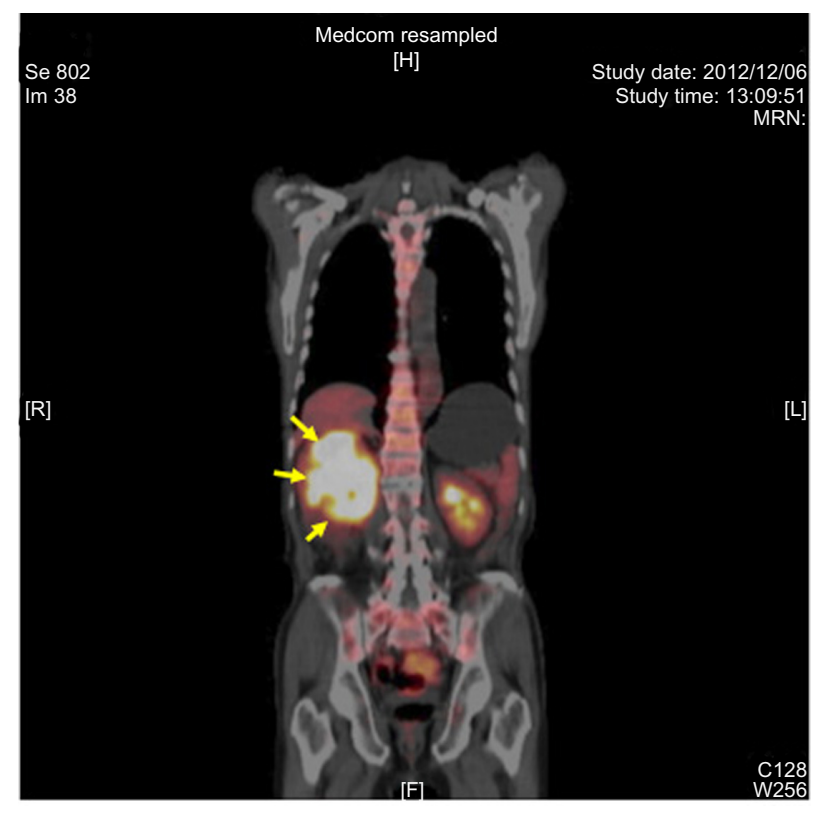

Figure 2 PET scan.

Notes: Scan shows accumulation of FDG in the right renal tumor (arrows). Maximum SVU is $>40$.

Abbreviations: PET, positron emission tomography; FDG, fluorine-18deoxyglucose; SUV, standardized uptake value.

sarcomatoid differentiation (cT4N1M0). It seemed to be difficult to safely perform radical nephrectomy, cavotomy with thrombectomy, and pancreatoduodenectomy. In comparison with the first-generation vascular endothelial growth factor receptor (VEGFR) tyrosine kinase inhibitors (TKIs) like sorafenib and sunitinib, axitinib is a more potent and selec-

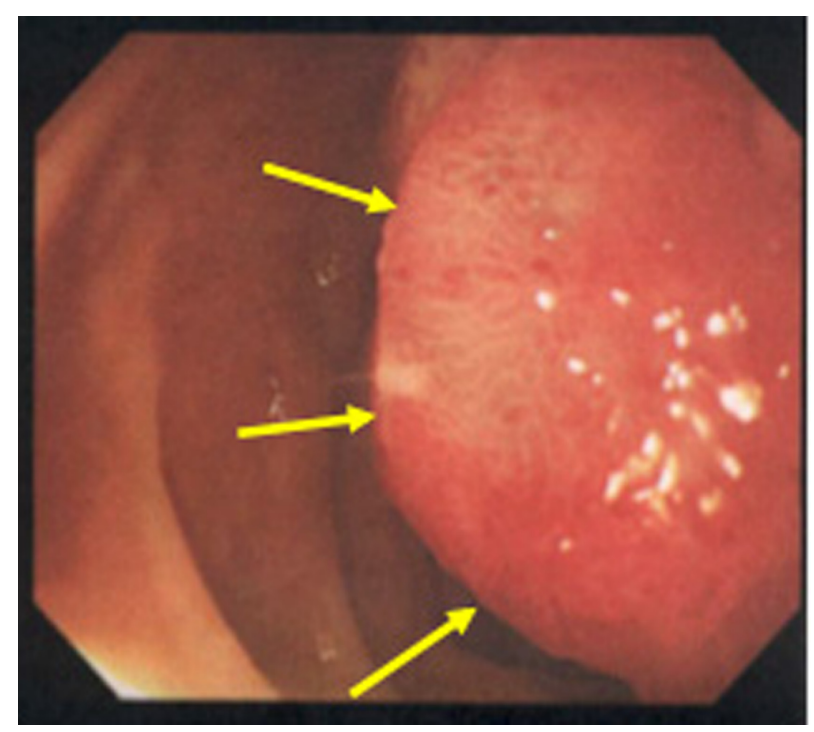

Figure 3 Esophagogastroduodenoscopy image.

Note: Esophagogastroduodenoscopy reveals a red sessile tumor protruding into the second part of the duodenum (arrows), corresponding to the findings of CT (Figure I).

Abbreviation: CT, computed tomography. 


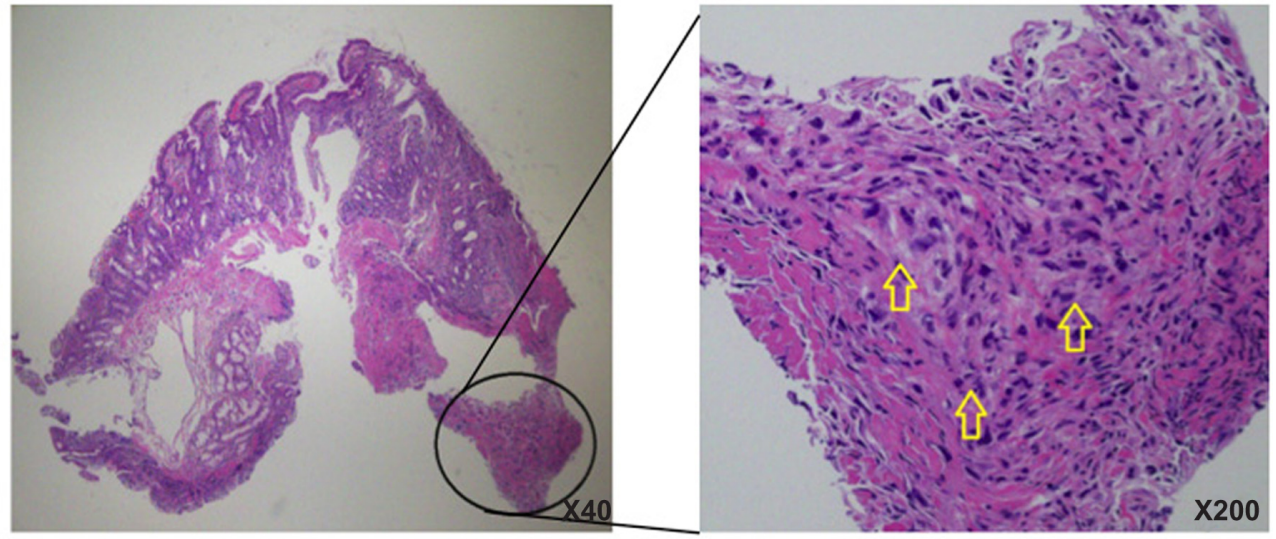

Figure 4 Histopathology of the biopsy specimen of duodenum.

Note: In the magnified encircled area, arrows show sarcomatoid tissue in the muscle layer of the duodenum.

tive second-generation VEGFR-TKI with better safety and tolerability, which has a higher affinity for the tyrosine kinase of VEGFR and a stronger inhibitory effect on its kinase activity with fewer serious adverse events, such as thrombocytopenia. ${ }^{4}$ At present, axitinib is generally recommended as a second-line therapy for advanced RCC, because the phase III axitinib versus sorafenib in advanced RCC (AXIS) trial demonstrated that axitinib treatment achieved longer progression-free survival than sorafenib in patients with metastatic RCC after failure of approved first-line systemic therapy, including sunitinib and cytokines. ${ }^{5}$

Our patient was unable to take food orally and had become emaciated. His classification was poor risk, according to the Memorial Sloan-Kettering Cancer Center (MSKCC) criteria. To decrease the tumor burden and improve the feasibility of surgery, we selected preoperative treatment with axitinib. If the patient showed a poor response to axitinib, we intended to perform palliative gastrojejunostomy to allow oral intake.

This study was conducted in accordance with the World Medical Association Inc's Declaration of Helsinki and was approved by the Institutional Review Board of the Ethical Committee of Dokkyo Medical University Hospital. In addition, the patient signed a consent form that was approved by our institutional Committee on Human Rights in Research. Axitinib administration (10 mg/day) was started in December 2012; it was scheduled to continue for 4-6 weeks until the patient underwent radical surgery, comprising nephrectomy, cavotomy with thrombectomy, and pancreatoduodenectomy.

During axitinib treatment, there were no adverse events $>$ grade 3 . After 2 weeks, the dose of axitinib was increased to $14 \mathrm{mg}$ /day; then, it was raised to $20 \mathrm{mg}$ /day after 2 more weeks. Attenuation of the tumor contrast uptake and enlargement of the duodenal lumen were observed on CT after 5 weeks of administration (Figure 5).

After 6 weeks of treatment, we decided to perform definitive surgery, and successfully carried out radical nephrectomy, cavotomy with thrombectomy, and pancreatoduodenectomy. The resected tumor was an invasive whitish and yellowish mass with partial necrosis. Pathological examination confirmed that the lesion was RCC with sarcomatoid differentiation with necrotic areas (pT4pN1M0, Fuhrman grade 4) (Figure 6). The pathological treatment effect of axitinib was grade 2 (two-thirds necrosis). The patient has been doing well since the operation. Western blotting and immunohistochemistry of the resected specimen was performed to detect

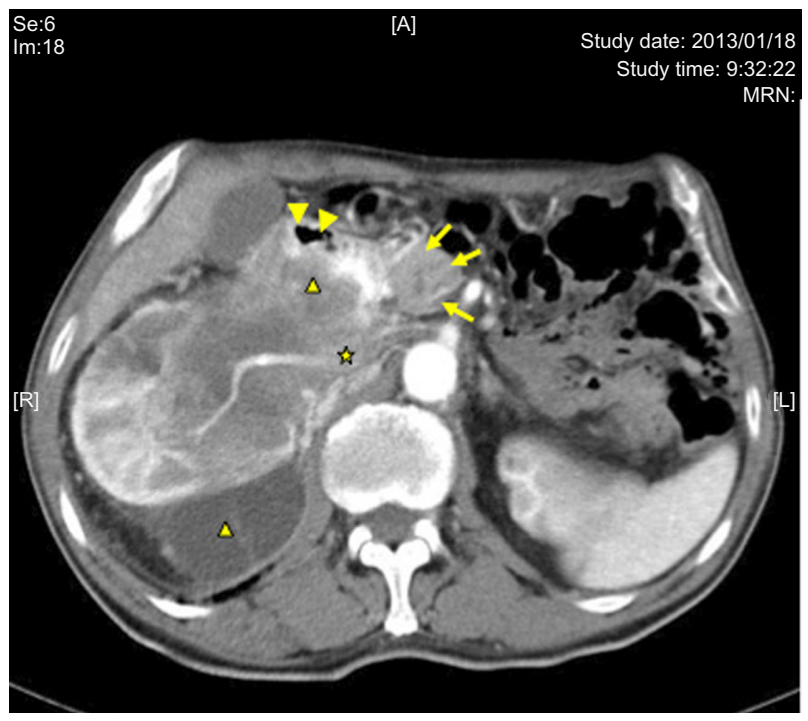

Figure 5 Enhanced CT at 5 weeks after starting treatment with axitinib. Notes: Tumor degeneration is observed (black triangle). The lumen of the second part of the duodenum (arrowheads) is wider, due to tumor shrinkage compared with the pretreatment state. Three arrows show the head of the pancreas, and the asterisk shows inferior vena cava.

Abbreviation: CT, computed tomography. 


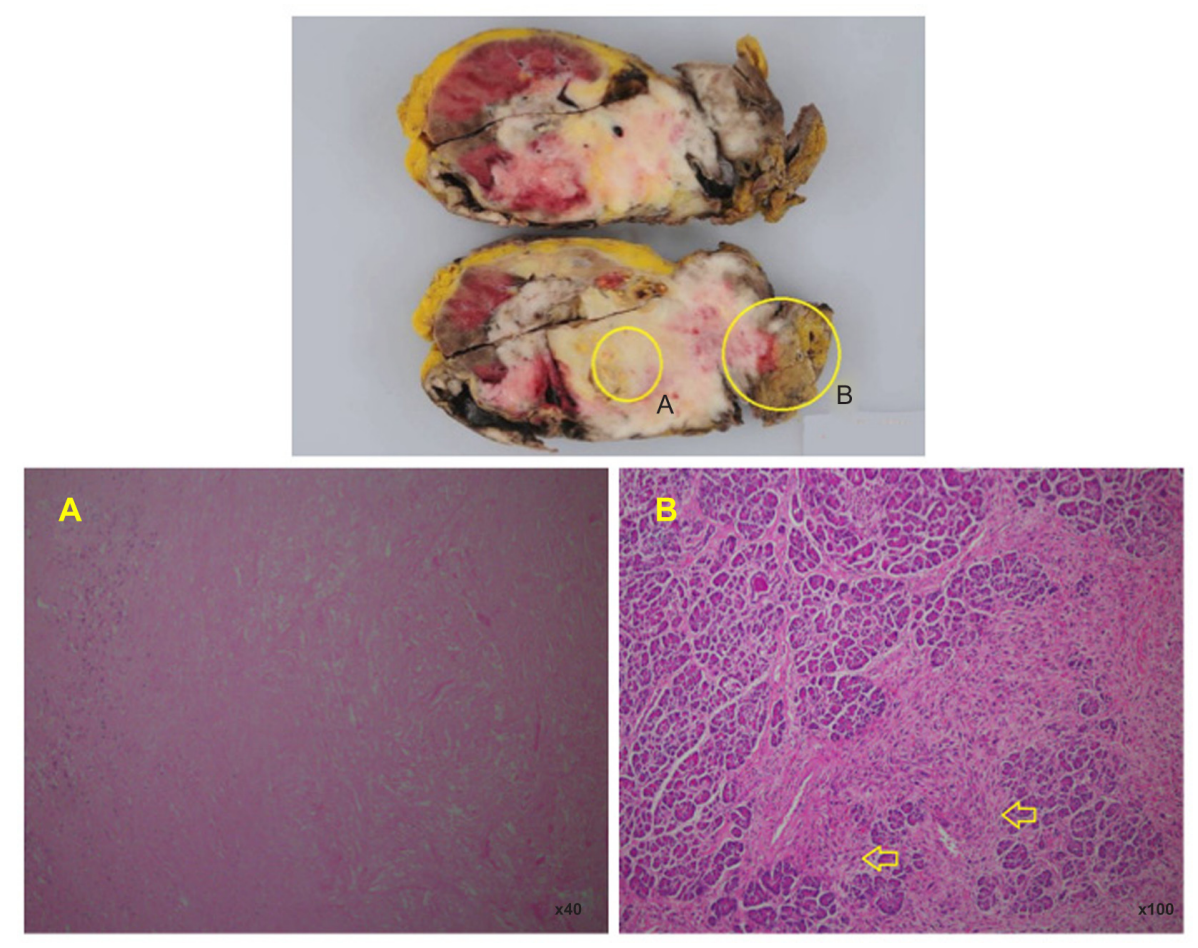

Figure 6 Macroscopic view of resected tissue.

Notes: (A) Shows necrotic tissue. (B) Shows sarcomatoid tissue invading pancreas (arrows).

phosphorylated Akt (Ser-473), using a rabbit antihuman antibody (PhosphoPlus ${ }^{\circledR}$ Akt (Ser-473) Antibody Kit; \#9270, Cell Signaling Technology, Inc., Danvers, MA, USA), according to the method described previously. ${ }^{6,7}$

These studies showed that expression of phosphorylated Akt (Ser-473) in the tumor was heterogeneous (Figures 7 and 8). Axitinib therapy (10 mg/day) was resumed because microscopic invasion was detected at the resection margin, and some of the tumor tissues showed viability and expression of phosphorylated Akt (Ser-473). The patient has been asymptomatic without apparent disease progression for 6 months after resuming treatment with axitinib.

\section{Discussion}

RCC with sarcomatoid differentiation is an uncommon tumor that displays characteristics derived from all subtypes of RCC and has the highest malignant potential, with a median overall survival time of only 6 months (range, approximately 0-68 months). ${ }^{8}$ Compared with conventional clear cell RCC (ccRCC), RCC with sarcomatoid differentiation is more invasive and refractory to treatment with a higher mortality. There are few effective agents for RCC with sarcomatoid differentiation. Although chemotherapy with gemcitabine, 5-FU, and doxorubicin seems to achieve slightly better results than cytokine therapy, ${ }^{9}$ systemic therapy is still challenging. Accordingly, achieving the curative resection of localized

\section{A}

p-Akt (Ser-473) (60 kDa)

Beta-actin (42 kDa)

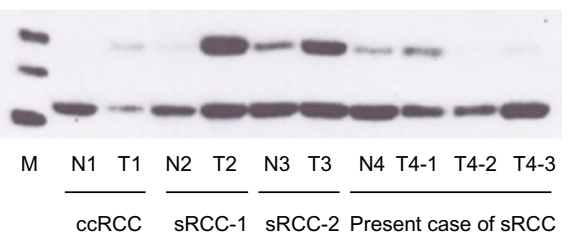

B

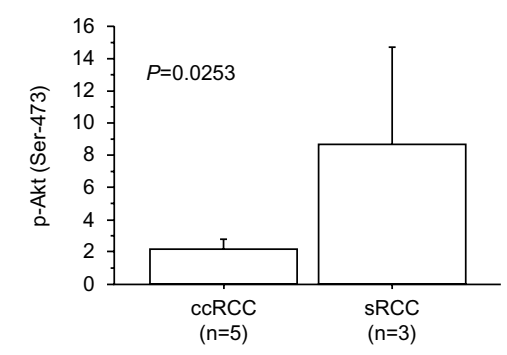

Figure 7 Expressions of phosphorylate Akt (Ser-473).

Notes: (A) Expression of phosphorylated Akt (Ser-473) $(60 \mathrm{kDa})$ and beta-actin $(42 \mathrm{kDa})$ proteins in the primary tumor tissues using Western blotting. Each number corresponds to a case number, representative case of ccRCC, other RCC with sarcomatoid differentiation (sRCCI and sRCC2), and present sRCC case. Patients of $\mathrm{cCRCC}$ and $\mathrm{sRCCI} / \mathrm{sRCC2}$ underwent nephrectomy before receiving any other therapy. Compared to ccRCC, tumor tissues of sRCCs showed higher expression of phosphorylated Akt (Ser-473). In the present sRCC case treated with presurgical axitinib, some tumor tissues of T4-2 and T4-3 showed an extremely lower expression of phosphorylated Akt (Ser-473) than T4-I, indicating that the T4-2 and T4-3 tissues were sensitive to axitinib. (B) Expression of phosphorylated Akt (Ser-473) in the primary tumors by densitometric analysis, as described previously. ${ }^{6}$ The data show the $95 \%$ confidence interval. Expression of phosphorylated Akt (Ser-473) was increased in $s R C C$ tumor tissue $(n=3$; mean $\pm S D=8.66 \pm 2.43)$ compared with those in $\operatorname{ccRCC}(n=5 ; 2.16 \pm 0.47 ; P=0.0253)$.

Abbreviations: $p$, phosphorylated; $M$, marker; $N$, non-tumor tissue; $n$, number of the patients analyzed; T, primary tumor tissue with metastatic lesions; RCC, renal cell carcinoma; ccRCC, clear cell renal cell carcinoma; sRCC, renal cell carcinoma with sarcomatoid differentiation; SD, standard deviation. 


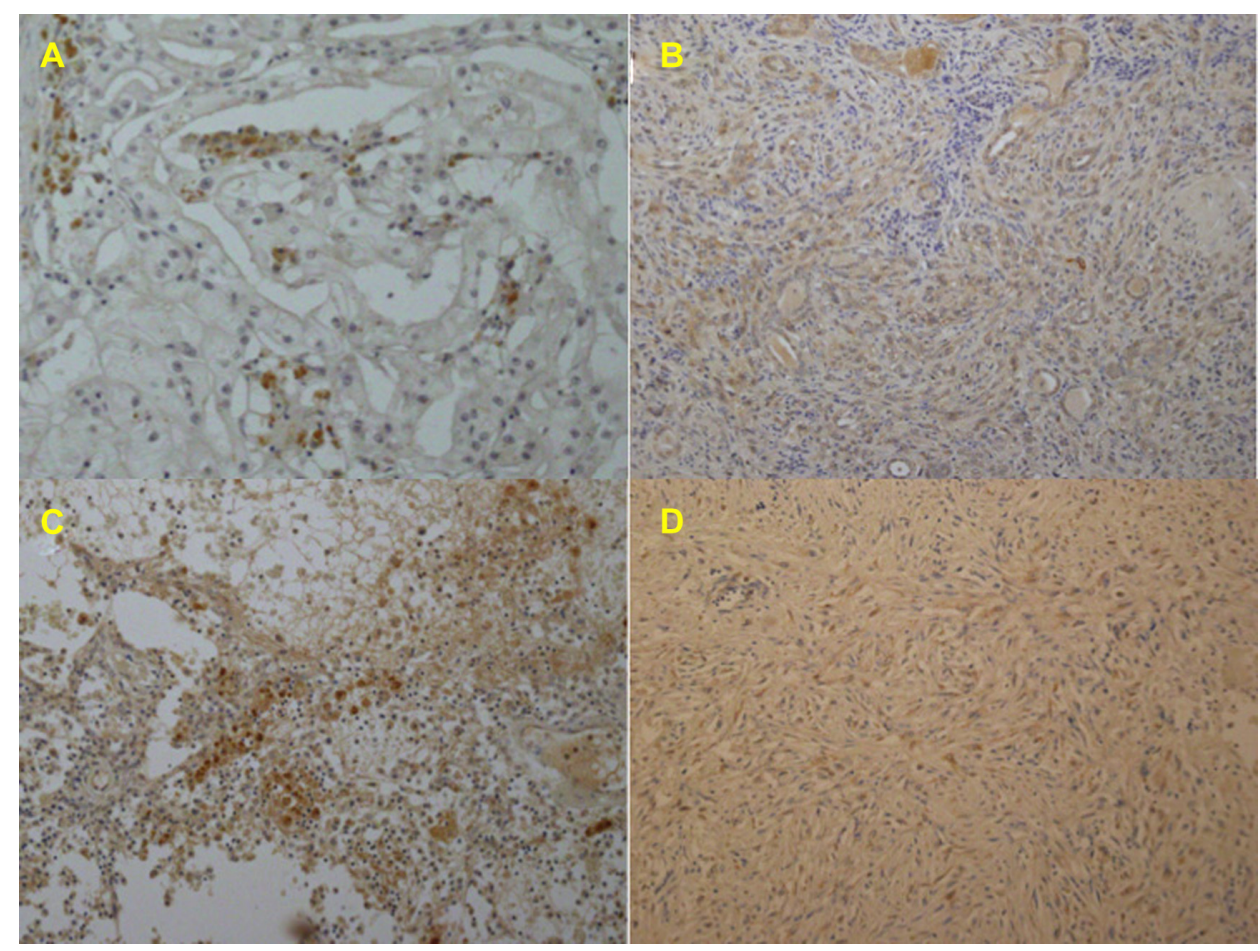

Figure 8 Representative immunohistochemistry in the primary tumor tissues for antiphosphorylated Akt (Ser-473) antibody.

Notes: Some of the tumor cells of $\operatorname{ccRCC}(\mathbf{A})$ showed brown staining in a membrane and cytoplasm, while many of the tumor cells did not. Most of the tumor cells of sRCCI (B) and sRCC2 (C) showed moderate to strong staining. In the present sRCC case treated with presurgical axitinib, some of the tumor cells of T4-I (D) showed weak reaction.

Abbreviations: ccRCC, clear cell renal cell carcinoma; sRCC, renal cell carcinoma with sarcomatoid differentiation.

or locally advanced RCC with sarcomatoid differentiation is very important.

Although the efficacy of cytoreductive nephrectomy has been confirmed, ${ }^{10}$ many patients who have locally advanced $\mathrm{RCC} / \mathrm{RCC}$ with sarcomatoid differentiation $(>\mathrm{cT} 3 \mathrm{~b}$ or $>\mathrm{cT}_{\text {any }} \mathrm{N} 1$ ) do not receive surgical treatment because of invasive disease, a poor performance status, or the high risk of complications. Several molecular-targeting agents (particularly first-generation TKIs) have been employed in the neoadjuvant setting to facilitate surgery by decreasing the tumor burden to reduce perioperative complications and bleedings. ${ }^{2,3}$ Sunitinib is currently the most extensively investigated agent and probably the most suitable choice for neoadjuvant therapy, but it only achieves a mean $10 \%$ decrease in the diameter of the primary tumor, and $20 \%-25 \%$ of patients show local progression during treatment. ${ }^{3}$ Thus, there is no evidence to support the use of molecular agents as routine neoadjuvant therapy for advanced RCC/RCC with sarcomatoid differentiation.

With regard to molecular-targeting therapy for metastatic RCC with sarcomatoid differentiation, Golshayan et al performed a retrospective study of 43 patients who had metastatic RCC with sarcomatoid differentiation and had undergone prior nephrectomy. ${ }^{11}$ Treatment with sunitinib, sorafenib, or bevacizumab, achieved a partial response of metastatic disease in a limited group of patients (19\%) who had underlying clear cell histology and a $>20 \%$ sarcomatoid component in the primary tumors, with median tumor shrinkage of $2 \%$, median time to progression of 5.3 months, and median overall survival of 11.8 months. These results suggested that VEGF-targeting therapy has only a modest effect on metastatic RCC with sarcomatoid differentiation. ${ }^{11}$ Molina et al also reviewed the outcome of systemic therapy for metastatic RCC with sarcomatoid differentiation in 63 patients (95\% with prior nephrectomy). ${ }^{12}$ They found a partial response to sunitinib in $12 \%$, and the median progression-free survival of patients treated with sunitinib was 4.4 months versus 2 months for other patients, suggesting that sunitinib achieved a modest response when employed for the treatment of metastatic RCC with sarcomatoid differentiation. ${ }^{12}$ These reports suggested that VEGF-targeting therapy, particularly sunitinib, might be suitable as systemic treatment for metastatic RCC with sarcomatoid differentiation. However, commonly reported grade 3/grade 4 toxicities associated with sunitinib ${ }^{13}$ include gastrointestinal disorders (diarrhea or anorexia) and hematological disorders (thrombocytopenia or neutropenia), which were a concern because the tumor directly invaded the duodenum and the inferior vena cava, 
and because our patient was unable to take food orally and had become emaciated with anemia and gastrointestinal bleeding.

Axitinib is a potent and selective second-generation VEGFR-TKI with better safety and tolerability than sunitinib, as well as a different adverse event profile with regard to hematological toxicities, such as thrombocytopenia. ${ }^{4}$ In the AXIS trial, axitinib achieved longer progression-free survival than sorafenib when used to treat metastatic RCC after failure of approved first-line systemic therapy, including sunitinib and cytokines. ${ }^{5}$ On the other hand, it has been recently reported that axitinib did not significantly increase progression-free survival in patients with treatment-naïve metastatic RCC (85\% with prior nephrectomy), compared with those treated with sorafenib $(90 \%$ with prior nephrectomy), but it did demonstrate the clinical activity and a manageable safety profile. ${ }^{14}$ At present, axitinib is generally recommended as a second-line therapy for advanced $\mathrm{RCC}$, but we chose axitinib as neoadjuvant therapy to decrease the tumor size and to improve the feasibility of surgery.

It has been reported that axitinib treatment can achieve extended disease control and symptom control in patients with metastases, ${ }^{4,5}$ and we found that the tumor burden was reduced without severe adverse events in the present case. Therefore, it might be argued that given the good response to preoperative axitinib therapy, it would have been preferable to continue axitinib for a longer period of time instead of performing surgery. However, RCC with sarcomatoid differentiation is an aggressive and invasive tumor, and our patient was MSKCC poor risk at the start of treatment. Thus, there was a risk that the tumor would show rapid progression or the patient's performance status would deteriorate during axitinib therapy, resulting in the optimal timing for surgery being missed.

Koie et al recently reported on a 73-year-old man who had clear cell RCC (cT2aN0M0 and MSKCC poor risk) with anemia, hypercalcemia, and poor performance status. ${ }^{15}$ Nephrectomy was performed without severe morbidity or perioperative complication after neoadjuvant axitinib therapy, ${ }^{15}$ which is a similar treatment strategy to ours and highlights the potential of preoperative axitinib therapy in this setting. Careful patient selection and meticulous surgical technique are essential in the treatment of patients with advanced RCC, and these points should be further emphasized in the era of targeted therapy.

The hyperactivation of phosphatidylinositol 3'kinase, serine/threonine kinase Akt, and the mammalian target of rapamycin pathway have been reported in a variety of human cancers, including RCC. ${ }^{16}$ We previously reported that tumors with higher levels of phosphorylated Akt (Ser-473) were resistant to interferon alpha with/without sorafenib therapy, and that patients whose tumors had higher levels of phosphorylated Akt (Ser-473) showed shorter overall survival. ${ }^{6}$ Similarly, Jonasch et al reported that an increase of phosphorylated Akt (Ser-473) on microarray analysis was associated with worse survival in patients receiving sorafenib with/without interferon alpha therapy. ${ }^{17}$ Therefore, we examined the expression of phosphorylated Akt (Ser-473) in the present patient. Western blotting and immunohistochemistry (Figures 7 and 8) of the nephrectomy specimen after axitinib therapy showed a heterogeneous reaction. Some tumor tissues (T4-2 and T4-3) showed extremely low expression of phosphorylated Akt (Ser-473) compared with other tissues (T4-1), indicating that some cancer cells were sensitive to axitinib and others were not. The present findings suggested that the efficacy of neoadjuvant molecular therapy combined with nephrectomy and/or metastasectomy should be reconsidered based on data obtained when the resected specimens are examined for expression of phosphorylated Akt (Ser-473) or other molecules, and this may shed new light on case selection and the prediction of the prognosis.

An investigation of the usefulness of axitinib for preoperative or neoadjuvant therapy in patients with locally advanced RCC is now ongoing. Since this is only a single case report about our experience with surgery after axitinib treatment, the results should be interpreted with consideration of such limitations and definite conclusions cannot be obtained. Further studies are needed to determine whether patients with locally advanced $\mathrm{RCC}(>\mathrm{cT} 3 \mathrm{~b}$ or $>\mathrm{cT}$ any $\mathrm{N} 1)$ can undergo surgical treatment with a better outcome after receiving neoadjuvant axitinib therapy. In such studies, we should also further analyze the outcome to assess the value of detecting phosphorylated Akt (Ser-473) as a surrogate marker in locally advanced RCC.

Obtaining such information will shed light on the clinical issue of whether tumor sensitivity to axitinib prior to cytoreductive nephrectomy is a useful marker for the choice of subsequent medical treatment. It is our hope that more patients with locally advanced RCC $(>\mathrm{cT} 3 \mathrm{~b}$ or $>\mathrm{cT}$ any $\mathrm{N} 1)$ will be able to undergo surgical treatment and achieve a better outcome after receiving preoperative therapy.

\section{Acknowledgment}

The authors are grateful to Hitomi Yamazaki for her excellent technique in Western blotting and immunohistochemical study. 


\section{Disclosure}

The authors report no conflicts of interest in this work.

\section{References}

1. Wagner B, Patard JJ, Méjean A, et al. Prognostic value of renal vein and inferior vena cava involvement in renal cell carcinoma. Eur Urol. 2009;55(2):452-459.

2. Harshman LC, Yu RJ, Allen GI, Srinivas S, Gill HS, Chung BI. Surgical outcomes and complications associated with presurgical tyrosine kinase inhibition for advanced renal cell carcinoma (RCC). Urol Oncol. 2013;31(3):379-385.

3. Schrader AJ, Steffens S, Schnoeller TJ, Schrader M, Kuczyk MA. Neoadjuvant therapy of renal cell carcinoma: a novel treatment option in the era of targeted therapy? Int J Urol. 2012;19(10):903-907.

4. Grünwald V, Merseburger AS. Axitinib for the treatment of patients with advanced metastatic renal cell carcinoma (mRCC) after failure of prior systemic treatment. Onco Targets Ther. 2012;5:111-117.

5. Rini B, Escudier B, Tomczak P, et al. Comparative effectiveness of axitinib versus sorafenib in advanced renal cell carcinoma (AXIS): a randomised phase 3 trial. Lancet. 2011;378(9807):1931-1939.

6. Furuya N, Kamai T, Shirataki H, et al. Serum interferon alpha receptor 2 mRNA may predict efficacy of interferon alpha with/without low-dose sorafenib for metastatic clear cell renal cell carcinoma. Cancer Immunol Immunother. 2011;60(6):793-808.

7. Horiguchi A, Oya M, Uchida A, Marumo K, Murai M. Elevated Akt activation and its impact on clinicopathological features of renal cell carcinoma. J Urol. 2003;16(2):710-713.

8. Keegan KA, Schupp CW, Chamie K, Hellenthal NJ, Evans CP, Koppie TM. Histopathology of surgically treated renal cell carcinoma: survival differences by subtype and stage. J Urol. 2012;188(2):391-397.
9. Shuch B, Bratslavsky G, Linehan WM, Srinivasan R. Sarcomatoid renal cell carcinoma: a comprehensive review of the biology and current treatment strategies. Oncologist. 2012;17(1):46-54.

10. Flanigan RC, Mickisch G, Sylvester R, Tangen C, Van Poppel H, Crawford ED. Cytoreductive nephrectomy in patients with metastatic renal cancer: a combined analysis. J Urol. 2004;171(3): 1071-1076.

11. Golshayan AR, George S, Heng DY, et al. Metastatic sarcomatoid renal cell carcinoma treated with vascular endothelial growth factor-targeted therapy. J Clin Oncol. 2009;27(2):235-241.

12. Molina AM, Tickoo SK, Ishill N, et al. Sarcomatoid-variant renal cell carcinoma. treatment outcome and survival in advanced disease. Am J Clin Oncol. 2011:34(5):454-459.

13. Motzer RJ, Huston TE, Tomczak P, et al. Sunitinib versus interferon alfa in metastatic renal-cell carcinoma. $N$ Engl J Med. 2007;356(2): $115-124$.

14. Hutson TE, Lesovoy V, Al-Shukri S, et al. Axitinib versus sorafenib as first-line therapy in patients with metastatic renal-cell carcinoma: a randomised open-label phase 3 trial. Lancet Oncol. 2013;14(13) 1287-1294.

15. Koie T, Ohyama C, Okamoto A, et al. Presurgical therapy with axitinib for advanced renal cell carcinoma: a case report. BMC Res Notes. 2013;6(1):484.

16. Abe H, Kamai T. Recent advances in the treatment of metastatic renal cell carcinoma. Int J Urol. 2013;20(10):944-955.

17. Jonasch E, Corn P, Pagliaro LC, et al. Upfront, randomized, phase 2 trial of sorafenib versus sorafenib and low-dose interferon alfa in patients with advanced renal cell carcinoma: clinical and biomarker analysis Cancer. 2010;116(1):57-65.
OncoTargets and Therapy

\section{Publish your work in this journal}

OncoTargets and Therapy is an international, peer-reviewed, open access journal focusing on the pathological basis of all cancers, potential targets for therapy and treatment protocols employed to improve the management of cancer patients. The journal also focuses on the impact of management programs and new therapeutic agents and protocols on

\section{Dovepress}

patient perspectives such as quality of life, adherence and satisfaction. The manuscript management system is completely online and includes a very quick and fair peer-review system, which is all easy to use. Visit http://www.dovepress.com/testimonials.php to read real quotes from published authors. 